\title{
Appetitive Memory with Survival Benefit Is Robust Across Aging in Drosophila
}

\author{
Ayako Tonoki, Mina Ogasawara, Zhihua Yu, and Motoyuki Itoh \\ Department of Biochemistry, Graduate School of Pharmaceutical Sciences, Chiba University, Chiba 260-8675, Japan
}

The formation of memory declines with advancing age. However, susceptibility to memory impairments depends on several factors, including the robustness of memory, the responsible neural circuits, and the internal state of aged individuals. How age-dependent changes in internal states and neural circuits affect memory formation remains unclear. Here, we show in Drosophila melanogaster that aged flies of both sexes form robust appetitive memory conditioned with nutritious sugar, which suppresses their high mortality rates during starvation. In contrast, aging impairs the formation of appetitive memory conditioned with non-nutritious sugar that lacks survival benefits for the flies. We found that aging enhanced the preference for nutritious sugar over non-nutritious sugar correlated with an age-dependent increase in the expression of Drosophila neuropeptide $F$, an ortholog of mammalian neuropeptide Y. Furthermore, a subset of dopaminergic neurons that signal the sweet taste of sugar decreases its function with aging, while a subset of dopaminergic neurons that signal the nutritional value of sugar maintains its function with age. Our results suggest that aging impairs the ability to form memories without survival benefits; however, the ability to form memories with survival benefits is maintained through age-dependent changes in the neural circuits and neuropeptides.

Key words: aging; appetitive memory; Drosophila; learning and memory; neuropeptide F

Significance Statement

The susceptibility to age-dependent memory impairments depends on the strength of the memory, changes in the responsible neurons, and internal states of aged individuals. How age-dependent changes in such internal states affect neural activity and memory formation remains unclear. We show in Drosophila melanogaster that aged flies of both sexes form robust appetitive memory conditioned with nutritious sugar, which has survival benefits for aged flies. In contrast, aging impairs the formation of appetitive memory conditioned with non-nutritious sugar that lacks survival benefits for the flies. Aging changes the neural circuits including dopamine neurons and neuropeptide F-expressing neurons, leading to the age-dependent impairment in memory with insufficient survival benefits and the preservation of the ability to form memory with survival benefits.

\section{Introduction}

Aging impairs memory formation; however, aging exerts differential effects across different types of memory. The susceptibility to age-dependent impairments of memory depends on the strength of the memory, changes in the responsible neurons, and internal states of aged individuals. Aging changes the individual's internal

Received Aug. 22, 2019; revised Jan. 14, 2020; accepted Jan. 15, 2020.

Author contributions: A.T., M.O., Z.Y., and M.I. designed research; A.T., M.O., and Z.Y. performed research; A.T., M.O., and Z.Y. analyzed data; A.T. and M.I. edited the paper; A.T. and M.I. wrote the paper.

This work was supported by the Ministry of Education, Culture, Sports, Science and Technology (MEXT) of Japan (Grants-in-Aid for Scientific Research on Innovative Areas 26115505, 16H01265, and 17H05736 to A.T.; Grant-in-Aid for Scientific Research 26830003 from MEXT to A.T.), the Narishige Neuroscience Research Foundation, the Takeda Science Foundation, and the Mochida Memorial Foundation for Medical and Pharmaceutical Research (A.T.). Fly stocks were provided by the Bloomington Drosophila Stock Center and Dr. Ronald L. Davis. We thank Sachiko Kinoshita for technical assistance.

The authors declare no competing financial interests.

Correspondence should be addressed to Ayako Tonoki at tonoki@chiba-u.jp.

https://doi.org/10.1523/JNEUROSCI.2045-19.2020

Copyright $@ 2020$ the authors states such as the response to starvation and satiety. How agedependent changes in such internal states affect neural activity and memory formation remains unclear.

Drosophila olfactory learning is ideally appropriate to address these questions, as flies that associate odors with electric shock or sugar form aversive or appetitive memories, respectively, and appetitive memory formation is significantly dependent on the internal states. Although aversive memory after a single training cycle conditioning is comparatively fragile and unstable over $24 \mathrm{~h}$ (Tully et al., 1994), appetitive conditioning after a single training cycle forms robust memory that is stable over $24 \mathrm{~h}$ (Tempel et al., 1983; Krashes and Waddell, 2008). A robust appetitive memory is formed only when flies have been starved and subsequently conditioned with nutritious sugars. The current working models suggest that two distinct subsets of dopaminergic neurons (DANs) in the protocerebral anterior medial (PAM) cluster signal sweet taste and the nutrient value of sugar, and these DANs are critical for the formation of short-term memory (STM) and long-term 
memory (LTM), respectively (Huetteroth et al., 2015; Yamagata et al., 2015). The DANs tile onto distinct lobes of the mushroom body (MB) neurons, which receive information from multiple primary sensory neurons and are critical for memory formation in the fly brain (Mao, 2009; Aso et al., 2014a,b). Furthermore, the robustness of appetitive memory is also affected by neuromodulatory systems such as Drosophila neuropeptide F (dNPF), an ortholog of mammalian neuropeptide Y (NPY) (Krashes et al., 2009).

Recent studies have shown that in Drosophila, aging decreases intermediate-term memory formation after single-cycle aversive conditioning (Tamura et al., 2003; Tonoki and Davis, 2012) and protein-synthesis-dependent LTM after multiple-cycle aversive conditioning with a rest between cycles (Mery, 2007; Tonoki and Davis, 2015). However, how aging might affect appetitive memory formation has not been previously investigated.

Here, we report that aged flies form robust appetitive memory when conditioned with nutritious sugars conveying survival benefits during starvation, but do not form appetitive memories when conditioned with non-nutritious sugars with insufficient survival benefits. Aging enhances the preference of nutritious sugar over non-nutritious sugar correlated with an age-dependent increase in $d N P F$ expression. Furthermore, a subset of DANs that signal nonnutritious sugar decreases its function with aging. Our results suggest that while aging impairs memory formation without survival benefits, it retains memory formation with survival benefits under food shortage, through the age-dependent changes in neural circuits including DANs and neuropeptides.

\section{Materials and Methods}

Fly strains. Flies were reared and crossed on standard medium at $25^{\circ} \mathrm{C}$ with $70 \%$ relative humidity in a $12 \mathrm{~h}$ light-dark cycle. Canton-S flies (except for Fig. $1 B$ ) or $w(C S 10)$ flies (see Fig. $1 B$ ) of mixed sex were used as wild-type strain in our experiments. The $w(C S 10)$ line is a Canton-S strain carrying a mutation in the white gene and was provided by Dr. Ronald L. Davis. Approximately 250 flies born in 2-3 d were raised in food bottles and transferred to fresh bottles every 3 or $4 \mathrm{~d}$ until reaching the appropriate age for each experiment. Flies carrying uas transgenes included UAS-mCD8-GFP (Lee and Luo, 1999) and UAS-dTrpA1 (Hamada et al., 2008). Gal4 driver lines, including dNPF-Gal4, R48B04, and R15A04, were obtained from the Bloomington stock center.

Learning and memory assay. Learning and memory assays were performed under dim red light at $23^{\circ} \mathrm{C}$ and $70 \%$ relative humidity. Appetitive olfactory memory experiments were performed as previously described (Cervantes-Sandoval and Davis, 2012). Briefly, flies of mixed sex were first food-deprived in $0.8 \%$ non-nutritive agar before training for 40 or $18 \mathrm{~h}$ in 10- and 30-d-old flies, respectively, except for specific additional conditions for each experiment. Approximately 50 starved flies were loaded into a training tube containing a dry filter paper previously soaked with water and exposed to the conditioned stimulus (CS-) odor for $2 \mathrm{~min}$ followed by $30 \mathrm{~s}$ of air. The flies were then transferred to a second tube containing a dry filter paper previously saturated with $2 \mathrm{M}$ sucrose, $3 \mathrm{~m}$ arabinose, or a mixture of $3 \mathrm{~m}$ arabinose with $2 \mathrm{~m}$ sorbitol solution and exposed for $2 \mathrm{~min}$ to a second CS + odor. For conditioning odors, we bubbled fresh air through 3-Octanol (Oct) and benzaldehyde (Benz) at concentrations of $0.12 \%$ and $0.08 \%$, respectively, in mineral oil. To measure immediate memory, we transferred the flies from a training tube directly into a T-maze where they were allowed $2 \mathrm{~min}$ to choose between two arms, one with the CS+ and the other with the CS - odor. To test memory performance $3 \mathrm{~h}$ after conditioning, the flies were kept in agar vials, at $23^{\circ} \mathrm{C}$, until memory performance was tested. To test memory performance $24 \mathrm{~h}$ after conditioning, flies were initially placed back into a food vial for $8 \mathrm{~h}$, following the conditioning, and then starved in agar vials until the memory test was conducted. For behavioral measurements, two groups of flies were trained simultaneously using two differ- ent odors as the CS + . The one-half performance index (PI) for each odor was calculated as follows: (number of flies that chose the CS+ minus the number that chose the CS - )/(number of flies that chose the CS- plus the number of flies that chose the CS+). The overall PI was then calculated as the average of the two one-half PIs for each odor. This method balances out naive odor biases.

PER assay. The PER assay was performed as previously described (Inagaki et al., 2012). Ten experimental flies were mounted into a chopped p200 tips to expose only the heads. The flies were tested if they show response to stepwise increasing concentration of sucrose or arabinose soaked in small parts of Kimwipe-paper. Only full extensions of the proboscis were counted. Flies that continuously responded to water or showed no response to $800 \mathrm{~mm}$ sucrose or arabinose, were excluded. We withdrew the paper soaked with sugar as soon as possible after touching it to the labelum, so that flies could not consume the sugar solution.

Two-choice assay. The two-choice preference assay was performed as previously described (Dus et al., 2011). Briefly, 50 male flies at the indicated age were first starved in vials with Kimwipe-paper wetted with $2 \mathrm{ml}$ of water. The flies were cold-anesthetized, transferred into 60 -well microtiter plates (60-well HLA terasaki plates with lids), and then given a choice between two kinds of sugar, sucrose and D-arabinose, with blue or red tasteless food dyes for $2 \mathrm{~h}$. The flies were scored by examining the color of their abdomen. The preference index was calculated as follows: [ number of flies that ate sucrose $+0.5 \times$ number of flies that ate both) - (number of flies that ate D-arabinose $+0.5 \times$ number of flies that ate both)]/(total number of flies that ate).

Feeding test. The feeding test was performed as described previously, with some modifications (Fujita and Tanimura, 2011). A group of 50 male flies was starved for $18 \mathrm{~h}$ in a vial with a Kimwipe-paper wetted with $2 \mathrm{ml}$ of water. Flies were then allowed to feed for $30 \mathrm{~min}$ on a $1.5 \mathrm{ml}$ agar gel in a vial with $1 \%(\mathrm{w} / \mathrm{v})$ blue dye and $100 \mathrm{~mm}$ concentration of each sugar. After feeding, the flies were immediately chilled in liquid nitrogen. To prevent eye pigment from interfering with the absorbance spectrum of the dye, fly bodies were separated from the heads and homogenized in $500 \mu \mathrm{l}$ of PBS buffer containing $0.01 \%$ Triton X-100. Samples were centrifuged twice, at 13,000 rpm for $25 \mathrm{~min}$. Absorbance of the supernatant was measured at $630 \mathrm{~nm}$ with a microplate reader (iMark, Bio-Rad). The absorbance measured for supernatants from flies fed with normal food was subtracted from the absorbance of the supernatant from flies fed the blue food. After determining the equivalent dye concentration of each fly homogenate using the linear fit of the standard curve $\left(R^{2}\right.$ was typically $>0.99$ ), the consumption of each fly was calculated.

Survival test. A group of 20 male flies at the indicated age was kept in a vial with $1.5 \mathrm{ml}$ of $0.8 \%$ non-nutritive agar containing sugar, at a concentration of $100 \mathrm{~mm}$, or no sugar, at $25^{\circ} \mathrm{C}$ and $70 \%$ relative humidity. The number of dead flies was counted every $12 \mathrm{~h}$. Ten repetitions were performed for each condition.

$q R T-P C R$. Total RNA was extracted from 50 fly heads or 10 fly bodies at the indicated age using TriPure Isolation Reagent (Roche), and reverse-transcription reactions were performed by using ReverTra Ace (Toyobo). Quantitative PCR was performed with SYBR Green (Power SYBR Green PCR Master Mix, Applied Biosystems) and an Applied Biosystems 7300 Real-Time PCR system. Expression levels were normalized against those of $r p 49$. A melting temperature analysis was used to confirm that each primer pair produced a single PCR product. The following

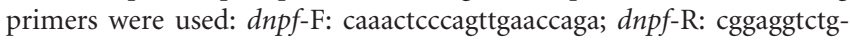
gagttggac; $n p f r-\mathrm{F}$ : cgtgtcgacaatatccataacg; $n p f r-\mathrm{R}$ : cacgaactgcaggctgtc; rp49-F: atcggttacggatcgaacaa; rp49-R: gacaatctccttgcgcttct.

Immunohistochemistry. We dissected and fixed male fly brains of the indicated genotype and age and incubated them overnight at $4{ }^{\circ} \mathrm{C}$, with antibody to mouse anti-nc82 (1:200; Developmental Studies Hybridoma Bank). Images were acquired using a Leica TCS SP8 confocal microscope. To quantify GFP signal for $d N P F$ expressing neurons processes, confocal $\mathrm{Z}$-stacks of each brain were obtained at a $1 \mu \mathrm{m}$ step size using identical laser power and scanning settings between brain samples. Image stacks were collapsed into a two-dimensional projection image for the figures and the fluorescence within the region of interest quantified using ImageJ software. Regions of interest (central brain) were delineated using the 


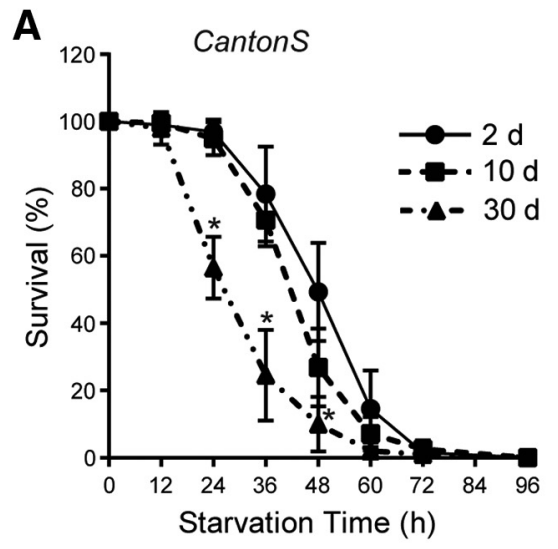

D

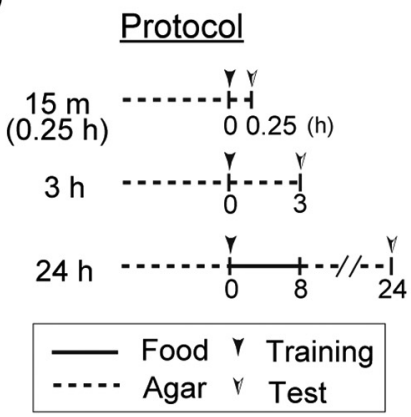

E
B
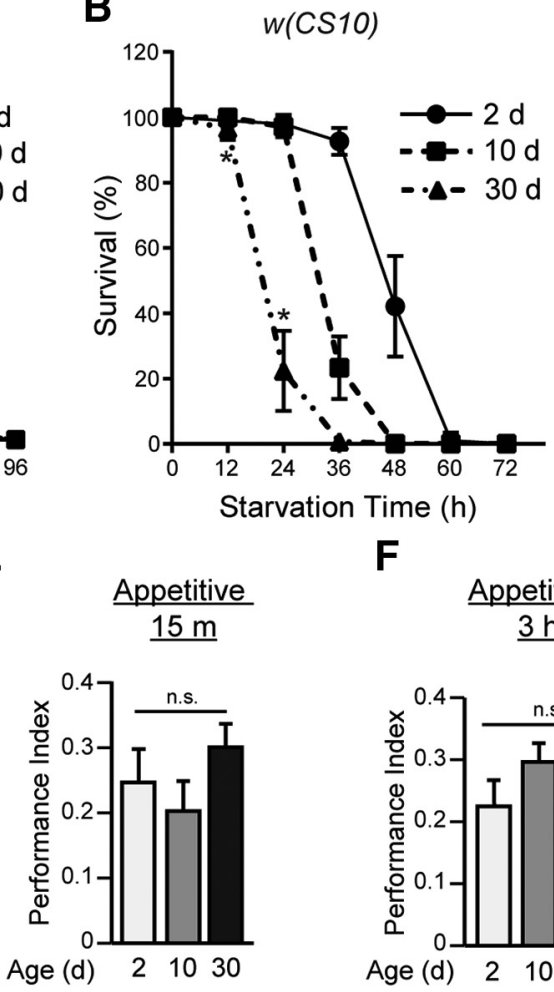

$\mathbf{F}$

Appetitive $\underline{3 \mathrm{~h}}$

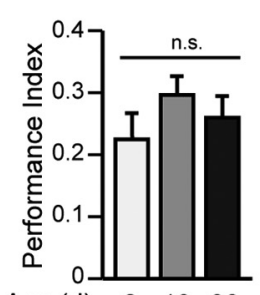

Age (d) $2 \quad 10 \quad 30$
C

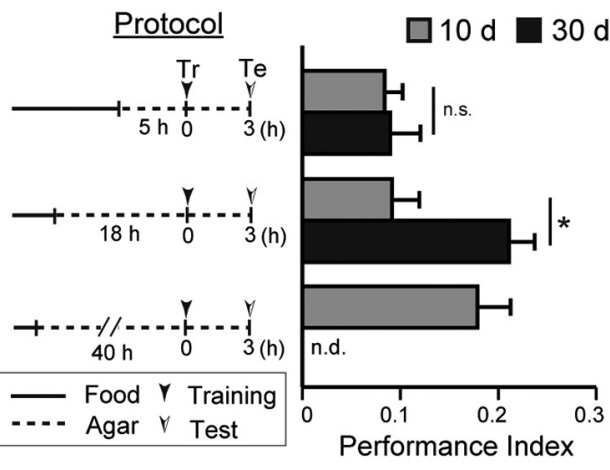

G

Appetitive $\underline{24 \mathrm{~h}}$

Figure 1. Aged flies form robust appetitive memory. A, Survival time course of Conton-S flies on $0.8 \%$ agar at 2, 10, or $30 \mathrm{~d}$ of age. The survival curve at $30 \mathrm{~d}$ of age is statistically different from that at 2 or $10 \mathrm{~d}$ of age for all time points of 24,36 , and $48 \mathrm{~h} . n=10$ for each data point. Data are mean \pm SD. $\boldsymbol{B}$, Survival time course of $w($ CS10) flies on $0.8 \%$ agar at 2,10 , or $30 \mathrm{~d}$ of age. The survival curve at $30 \mathrm{~d}$ of age is statistically different from that at 2 - or $10 \mathrm{~d}$ of age for all time points of 24 and $36 \mathrm{~h}$. $n=12,11$, and $12 \mathrm{for} 2 \mathrm{~d}, 10 \mathrm{~d}$, and $30 \mathrm{~d}$ data, respectively. Data are shown as mean \pm SD. C, Longer food deprivation time interval is required for the behavioral expression of appetitive memory in young flies ( $10 \mathrm{~d}$ ) compared with aged flies ( $30 \mathrm{~d}$ ). Three-hour appetitive memory following appetitive conditioning with sucrose after food deprivation of 5,18 , and $40 \mathrm{~h}$ in Canton- $S$ flies at 10 or $30 \mathrm{~d}$ of age. $n=$ 16 and 15 for 10 and $30 \mathrm{~d}$ data, respectively. D, Feeding protocols for appetitive memory. Flies experience food deprivation for 40 and $18 \mathrm{~h}$ in the 10 - and 30 - $\mathrm{d}$-old flies, respectively, before training and between training and testing, with the exception of a feeding $8 \mathrm{~h}$ after training, for testing at $24 \mathrm{~h}$. $\boldsymbol{E}$, Fifteen-minute performance index after appetitive conditioning with sucrose was unchanged across age groups in Canton-S wild-type flies. $n=12,12$, and 13 for 2,10 , and 30 d data, respectively. $\boldsymbol{F}$, Three-hour performance index after appetitive conditioning with sucrose was unchanged across age groups in Canton-S wild-type flies. $n=12,23$, and 11 for 2, 10, and $30 \mathrm{~d}$ data, respectively. $\mathbf{G}, 24 \mathrm{~h}$ performance index after appetitive conditioning with sucrose was unchanged across age groups in Canton-S wild-type flies. $n=24,12$, and 12 for 2,10 , and 30 d data, respectively. n.d., No data; n.s., not significant. Data are shown as mean \pm SEM for $\boldsymbol{C}$ and $\boldsymbol{E}-\boldsymbol{G}$.

nc82 signal and the area and mean fluorescence intensity in each region was measured using ImageJ.

Experimental design and statistical analysis. Analyses of the data were performed with Prism (GraphPad) software using appropriate ANOVAs to make comparisons among different groups. Significant effects were determined using a Tukey's post hoc test. We applied two-way ANOVA for Figures $2 A, C, F, 3 B-D$, and $4 B, C$, and one-way ANOVA for Figures $1 A, B, E-G$ and $3 H$. Unpaired Student's $t$ tests were used for two-group comparisons. We applied $t$ tests for Figures $1 C, 2 D, E$, and $3 G$. The comparisons were considered to be statistically significant when $p<0.05$. Memory scores were displayed as mean \pm SEM (Figs. $1 C, E-G, 2 A$, $4 B, C$ ). Other data including survival data, PER assay data, and qRT-PCR are displayed as mean $\pm \mathrm{SD}$ (Figs. $1 A, B, 2 B-F, 3 B-D, G, H$ ).

\section{Results}

\section{Aged flies form robust appetitive memory}

To examine the appetitive memory formation across the Drosophila lifespan, we assayed memory after appetitive olfactory conditioning in Canton-S wild-type flies of several different age groups. Considering that the formation of appetitive memory is observed in the presence of starvation, we verified the sensitivity to starvation in both young and aged flies by housing flies in food vials with $0.8 \%$ agarose. Most young flies at 2 or $10 \mathrm{~d}$ of age survived on agarose for at least $24 \mathrm{~h}$, whereas $>40 \%$ of the aged flies (30-d-old) were dead within $24 \mathrm{~h}$ (one-way ANOVA followed by post hoc Tukey's multiple-comparisons test at each star- vation time. $12 \mathrm{~h}: F_{(2,27)}=0.5963, p=0.5579 ; 24 \mathrm{~h}: F_{(2,27)}=128$, $p<0.0001$, post hoc Tukey's multiple-comparisons test, ${ }^{\star} p<$ $0.0001,2 \mathrm{~d}$ vs 30 and $10 \mathrm{~d}$ vs $30 \mathrm{~d} ; 36 \mathrm{~h}: F_{(2,27)}=57.28, p<0.0001$, post hoc Tukey's multiple-comparisons test, ${ }^{\star} p<0.0001,2 \mathrm{~d}$ vs 30 and $10 \mathrm{~d}$ vs $30 \mathrm{~d} ; 48 \mathrm{~h}: F_{(2,27)}=28.32, p<0.0001$, post hoc Tukey's multiple-comparisons test, ${ }^{\star} p<0.0001,2 \mathrm{~d}$ vs $30 \mathrm{~d} ; 60 \mathrm{~h}: F_{(2,27)}=$ $\left.7.541, p=0.0025 ; 72 \mathrm{~h}: F_{(2,27)}=0.7708, p=0.4725\right)($ Fig. $1 A)$, consistent with the results of the previous studies showing high sensitivity to starvation in aged flies (Lin et al., 1998). To confirm the age-dependent increase in starvation sensitivity, we examined the sensitivity to starvation in white control flies $(w(C S 10))$ (Fig. $1 B$ ). Aged flies of $w(C S 10)$ also showed high sensitivity to starvation (one-way ANOVA followed by post hoc Tukey's multiplecomparisons test at each starvation time. $12 \mathrm{~h}: F_{(2,32)}=6.989, p=$ 0.0030 , post hoc Tukey's multiple-comparisons test, $p=0.0357$ for $2 \mathrm{~d}$ vs $30 \mathrm{~d}$ and $p=0.0029$ for $10 \mathrm{~d}$ vs $30 \mathrm{~d} ; 24 \mathrm{~h}: F_{(2,32)}=387.9$, $p<0.0001$, post hoc Tukey's multiple-comparisons test, ${ }^{\star} p<$ $0.0001,2 \mathrm{~d}$ vs 30 and $10 \mathrm{~d}$ vs $30 \mathrm{~d}$ ), although the starvation sensitivity was higher in $w(C S 10)$ flies than in Canton-S flies.

Considering that the presence and extent of starvation in flies enables the formation of appetitive memory, we examined the duration of food deprivation that is required for effective appetitive memory performance in each of the Canton-S fly age groups. Flies were starved for 5,18 , or $40 \mathrm{~h}$ before conditioning, and 
memory performance was assayed $3 \mathrm{~h}$ after conditioning (Fig. $1 C)$. Starvation for $5 \mathrm{~h}$ before conditioning presented poor appetitive memory formation in both young and aged flies; however, starvation for $18 \mathrm{~h}$ before conditioning, when $20-30 \%$ of aged flies were dead but most of the young flies were alive, presented robust appetitive memory in aged flies, but poor appetitive memory formation in young flies $\left(5 \mathrm{~h}: t_{(29)}=0.1599, p=\right.$ $0.8740 ; 18$ h: $t_{(29)}=3.168,{ }^{*} p=0.0036$, unpaired $t$ test). Starvation for $40 \mathrm{~h}$ before conditioning, when $20-30 \%$ of young flies and most of the aged flies were dead, presented robust appetitive memory in young flies (Fig. $1 A, C$ ). These data indicate that 40 and $18 \mathrm{~h}$ of starvation are required in young flies and aged flies, respectively, to present a robust appetitive memory, suggesting that an age-dependent change in the sensitivity to starvation is also associated with appetitive memory formation.

We assayed memory formation and persistence after appetitive olfactory conditioning in Canton-S flies in different age groups. Flies at 2- or $10 \mathrm{~d}$ of age were starved before training for $40 \mathrm{~h}$, while the 30 -d-old flies were starved for $18 \mathrm{~h}$, and memory was measured at $15 \mathrm{~min}, 3 \mathrm{~h}$, and $24 \mathrm{~h}$ after appetitive conditioning (Fig. 1D). Although the previous reports have shown that aging impairs intermediate-term memory measured at 1 or $3 \mathrm{~h}$ after aversive conditioning (Tamura et al., 2003; Tonoki and Davis, 2012), significant change in the formation or retention of appetitive memory was not observed across the age groups when the starvation level was not different between young and aged flies $(15 \mathrm{~min}$ : $F_{(2,34)}=1.228, p=0.3056,3 \mathrm{~h}: F_{(2,34)}=2.593, p=0.0865,24 \mathrm{~h}:$ $F_{(2,45)}=0.02113, p=0.9791$, one-way ANOVA) (Fig. $\left.1 E-G\right)$.

\section{Robust formation of appetitive memory in aged flies requires nutritious sugar}

The recent studies have revealed that the non-nutritious sugar, arabinose, which has a sweet taste without nutrients, induces weak memory formation; however, robust memories are formed when arabinose is combined with sorbitol, a nutritious sugar that lacks sweetness (Burke and Waddell, 2011; Fujita and Tanimura, 2011). This prompted us to examine whether robust formation of appetitive memory in aged flies requires the presence of nutrients. We starved young flies for $40 \mathrm{~h}$ and aged flies for $18 \mathrm{~h}$, respectively, conditioned the flies using sucrose, arabinose, sorbitol, or sorbitol-supplemented arabinose as reinforcement, and measured the performance of appetitive memory $3 \mathrm{~h}$ after conditioning, in young and aged flies. The nutritious sugar sucrose or the sorbitol-supplemented arabinose formed robust memories in both the 10- and 30-d-old flies (Fig. 2A). Memories were also formed with the non-nutritious sugar arabinose in 10-d-old flies, although not in the 30-d-old flies (two-way ANOVA followed by post hoc Tukey's multiple-comparisons test. $F_{(1,59)}=9.445, p=$ 0.0032 , post hoc Tukey's multiple-comparisons test, sucrose: $p>$ $0.9999,10 \mathrm{~d}$ vs $30 \mathrm{~d}$; arabinose: ${ }^{\star} p=0.0225,10 \mathrm{~d}$ vs $30 \mathrm{~d}$; arabinose + sorbitol: $p=0.4510,10 \mathrm{~d}$ vs $30 \mathrm{~d}$ ) (Fig. $2 A)$. As shown in the previous experiments using young flies (Burke and Waddell, 2011), appetitive memory was not formed when aged flies were conditioned with sorbitol alone [performance index $(\mathrm{PI})=$ $0.1123 \pm 0.037, n=8]$. The PI score was insignificantly different from zero ( $p=0.0547$, Wilcoxon's signed-rank test), suggesting that both young and aged flies ingested insufficient amounts of sorbitol to form memory when sorbitol was presented during conditioning.

To verify the relative nutritious value of each sugar in young and aged flies, we measured the survival rate of 10- and 30-d-old flies by housing flies in vials containing sucrose, arabinose, and sorbitol-supplemented arabinose. Consistent with the previous studies (Burke and Waddell, 2011; Fujita and Tanimura, 2011), most flies housed on sucrose were alive for $72 \mathrm{~h}$, while $\sim 70 \%$ of both 10- and 30-d-old flies housed on agar or arabinose were dead by that time (Fig. 2B). Although aged flies housed on agar or arabinose died earlier than young flies (two-way ANOVA followed by post hoc Tukey's multiple-comparisons test. $F_{(1,72)}=$ 296.8, $p<0.0001$, post hoc Tukey's multiple-comparisons test, Sucrose: $p>0.9999,10$ d vs 30 d; Agar: ${ }^{* *} p<0.0001,10$ d vs 30 d; Arabinose: ${ }^{* *} p<0.0001,10 \mathrm{~d}$ vs $30 \mathrm{~d}$; arabinose + sorbitol: ${ }^{*} p=$ $0.0022,10 \mathrm{~d}$ vs $30 \mathrm{~d})$, their survival on arabinose supplemented with sorbitol, at $36 \mathrm{~h}$, was similar to their survival on sucrose $\left(F_{(3,72)}=\right.$ $123, p<0.0001$, post hoc Tukey's multiple-comparisons test, ${ }^{* *} p<$ 0.0001 , arabinose $30 \mathrm{~d}$ vs arabinose + sorbitol $30 \mathrm{~d}$ ) (gray arrow in Fig. $2 B, C$ ), suggesting that the low survival rate on agar or the nonnutritious sugar arabinose in aged flies was partially rescued by the addition of nutrients. These data suggested that the survival rate is coincidentally consistent with the performance of appetitive memory, suggesting that robust appetitive memory in aged flies requires nutritious sugar with survival benefits.

We next asked whether the age-dependent decrease in performance conditioned with arabinose was due to a low sensitivity to arabinose in the aged flies. To verify sugar sensitivity, we presented a drop of solution containing sugar to the labelum, where the gustatory receptor neurons are located. When flies detect sugar as favorable, they will extend their proboscis, a reaction known as the proboscis extension reflex (PER). A previous report shows that sugar sensitivity to sucrose is increased when young flies are starved on agar (Inagaki et al., 2012). When we compared PER dose-response curves against sucrose and arabinose in the starved state between young and aged flies (40 or $18 \mathrm{~h}$ of starvation for young and aged flies, respectively), significant difference was not observed (sucrose: $6.25 \mathrm{mM}: t_{(4)}=1.342, p=0.2508 ; 12.5$ $\mathrm{mM}: t_{(4)}=0.6124, p=0.5734 ; 25 \mathrm{mM}: t_{(4)}=1.061, p=0.3486 ; 50$ $\mathrm{mm}: t_{(4)}=1.604, p=0.1841 ; 100 \mathrm{~mm}: t_{(4)}=1.061, p=0.3486$; $200 \mathrm{mM}: t_{(4)}=1, p=0.3739$, unpaired $t$ test, arabinose: $6.25 \mathrm{~mm}$ : $t_{(8)}=0, p>0.9999 ; 12.5$ mM: $t_{(8)}=0.2182, p=0.8327 ; 25 \mathrm{mM}:$ $t_{(8)}=0.5852, p=0.5745 ; 50 \mathrm{~mm}: t_{(8)}=0.7845, p=0.4554 ; 100$ mM: $t_{(8)}=0.6667, p=0.5237 ; 200 \mathrm{mM}: t_{(8)}=1.291, p=0.2328$; $400 \mathrm{~mm}: t_{(8)}=0.6667, p=0.5237$, unpaired $t$ test), indicating that aging does not affect sugar sensitivity when the flies are starved to the same degree (Fig. 2D,E).

A reduction in the ingested amount of the non-nutritious sugar arabinose during conditioning could also lead to weak memory formation conditioned with arabinose in aged flies. To address this possibility, we measured the food ingestion of sucrose or arabinose in young and aged starved flies. Starved flies were allowed to feed on agar medium containing sucrose or arabinose for $30 \mathrm{~min}$ (Fujita and Tanimura, 2011). The amount of sucrose or arabinose ingested was insignificantly different between young and aged flies (two-way ANOVA followed by post hoc Tukey's multiple-comparisons test. $F_{(1,24)}=8.937, p=$ 0.0064 , post hoc Tukey's multiple-comparisons test, sucrose: $p=$ 0.2764 , $10 \mathrm{~d}$ vs $30 \mathrm{~d}$; arabinose: $p=0.0907,10 \mathrm{~d}$ vs $30 \mathrm{~d}$; No sugar: $p>0.9999,10 \mathrm{~d}$ vs $30 \mathrm{~d}$ ) (Fig. $2 F$ ). Together, these data indicate that aging decreases memory formation conditioned with arabinose, without age-dependent changes in sugar sensitivity or the ingested amount of each sugar.

\section{Aging modulates $d N P F$ pathway regulating nutritious sugar preference}

We next hypothesized that aging changes preferences regarding sugar quality. To assay sugar quality preference, we performed a two-choice preference assay in both young and aged flies (Fig. 
A
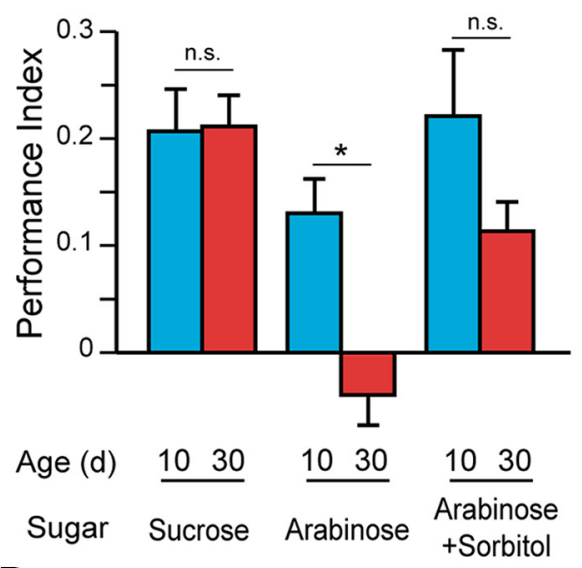

D

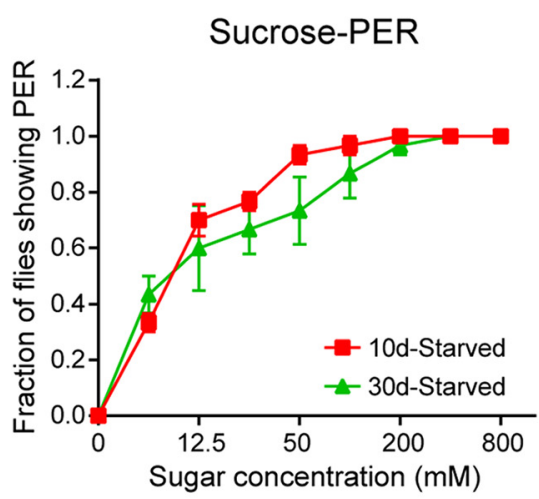

B

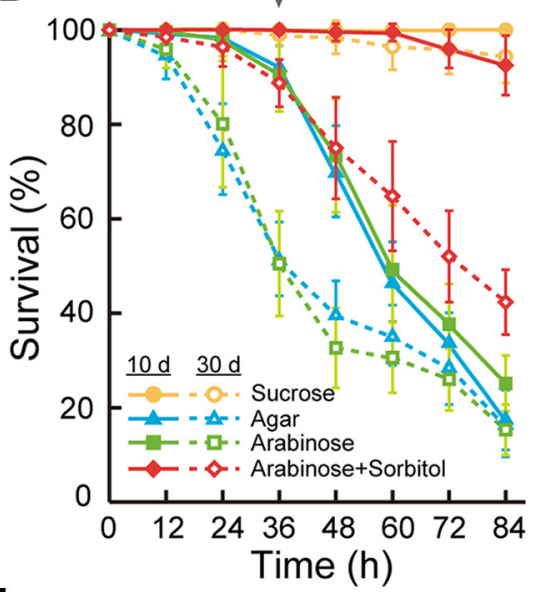

E

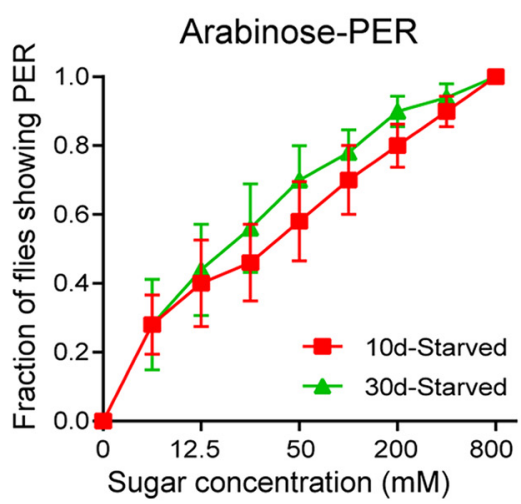

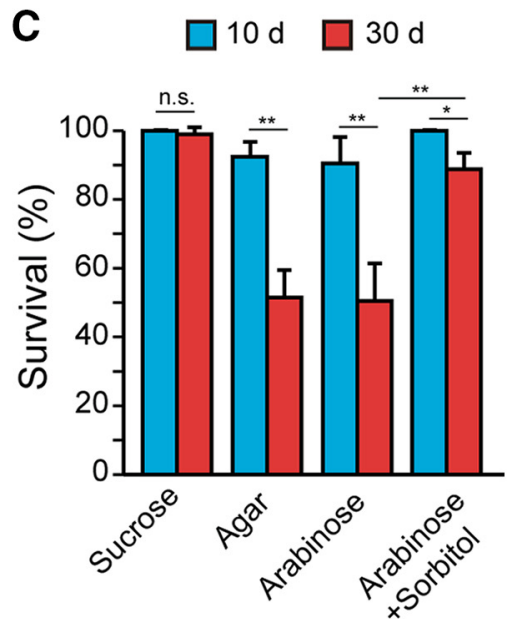

$\mathbf{F}$

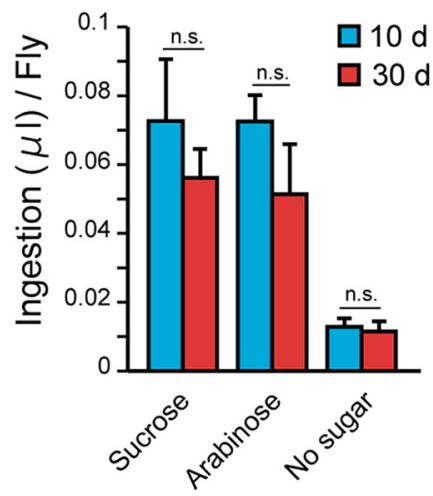

Figure 2. Behavioral expression of appetitive memory in aged flies requires nutritious sugar. $\boldsymbol{A}$, Three-hour PI after conditioning with the non-nutritious sugar arabinose was significantly reduced in 30-d-old flies compared with 10-d-old flies. Three-hour PI with sucrose or sorbitol-supplemented arabinose in the aged flies was statistically indistinguishable from young flies. $n=8,8,8,13,14$, and 14 for sucrose-10d, arabinose-10d, arabinose + sorbitol-10d, sucrose-30d, arabinose-30d, and arabinose + sorbitol-30d, respectively. n.s.: not significant. Data are shown as mean \pm SEM. $\boldsymbol{B}$, Survival time course of flies at 10 or $30 \mathrm{~d}$ of age, supplied with sugar at $100 \mathrm{~mm}$. Flies were supplied with agar containing sucrose, arabinose, or arabinose supplemented with sorbitol. $n=10$ for each data point. Data are shown as mean \pm SD. $\boldsymbol{C}$, The 36 -h time point is extracted from the graph of $\boldsymbol{B}$. Survival rate on agar or non-nutritious sugar arabinose is low in aged flies. The low survival rate on non-nutritious sugar arabinose in aged flies is recovered by addition of sorbitol. n.s.: not significant. Data are shown as mean \pm SD. D, Sensitivity of nutritious sugar sucrose in Canton-S flies in starved state is not significantly different between 10- and 30-d-old flies. Average fraction of flies showing PER to various concentrations of sucrose is shown. $n=3$ for each experimental group. Data are shown as mean \pm SD. $E$, Sensitivity of non-nutritious sugar arabinose in Canton-S flies in starved-state is not significantly different between 10- and 30-d-old flies. Average fraction of flies showing PER to various concentrations of arabinose is shown. $n=$ 5 for each experimental group. Data are shown as mean \pm SD. $F$, Amount of sugar intake (100 mm sucrose or $100 \mathrm{~mm}$ arabinose) over 30 min measured by the colorimetric method using a blue food dye in flies at 10 or $30 \mathrm{~d}$ of age. The amount of sugar intake is not significantly different between young and aged flies. $n=5$ for each data point. Data are shown as mean \pm SD. n.s., Not significant.

$3 A, B)$. Flies can select nutritious sugars over non-nutritious sugars after periods of food deprivation in a two-choice preference assay (Tanimura et al., 1988). These tested flies chose a more concentrated non-nutritious sugar, arabinose, over a less concentrated nutritious sugar, sucrose, but developed a preference for the nutritious sugar when the concentration was increased, as previously shown (Dus et al., 2011). We found that aged flies showed a stronger preference for the nutritious sugar sucrose over the non-nutritious sugar arabinose compared with young flies, when the sucrose-to-arabinose ratio was 1:10 - 3:20 (twoway ANOVA followed by post hoc Tukey's multiple-comparisons test. $F_{(1,22)}=44.4, p<0.0001$, post hoc Tukey's multiplecomparisons test, $1 \mathrm{~mm}: p>0.9999,10 \mathrm{~d}$ vs $30 \mathrm{~d} ; 20 \mathrm{~mm}:{ }^{*} p=$ 0.0002, $10 \mathrm{~d}$ vs $30 \mathrm{~d} ; 30 \mathrm{~mm}:{ }^{\star} p<0.0001,10 \mathrm{~d}$ vs $30 \mathrm{~d} ; 100 \mathrm{~mm}$ : $p>0.9999)$ (Fig. 3B).

The robustness of appetitive memory is known to be modulated by neuromodulatory systems such as $d N P F$, an ortholog of mammalian NPY (Krashes et al., 2009). Sensitivity to sugar is also modulated by $d N P F$ (Inagaki et al., 2014). NPY stimulates foodseeking behavior in mammals, and NPY levels are increased in the neurons in the arcuate nucleus of the hypothalamus in food-deprived rats (Sahu et al., 1988). To examine whether the expression levels of $d N P F$ changed across aging, we performed a qRT-PCR analysis of $d N P F$ and the $d N P F$ receptor ( $d N P F R$ ) in fly heads and bodies at 10 and $30 \mathrm{~d}$ of age. The expression of $d N P F$ and $d N P F R$ was observed mainly in the head (Fig. $3 C, D$ ). Interestingly, the expression of $d N P F$ was significantly upregulated in aged fly heads (two-way ANOVA followed by post hoc Tukey's multiple-comparisons test. $F_{(1,8)}=12.67, p=0.0074$, post hoc Tukey's multiple-comparisons test, head: ${ }^{\star} p=0.0039,10 \mathrm{~d}$ vs $30 \mathrm{~d}$ ) (Fig. $3 C$ ), although the expression of $d N P F R$ did not change with aging (two-way ANOVA. $F_{(1,8)}=0.4577, p=0.5178$ ) (Fig. 3D).

Subsequently, we examined the age-dependent changes in the projection pattern of $d N P F$ in the brain. As shown previously (Wen et al., 2005; Krashes et al., 2009), dNPF expression labeled with a $d N P F$ promoter-Gal4 (dNPF-Gal4) driven UAS-mCD8:: 
A

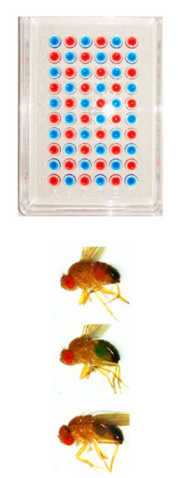

$\mathbf{E}$

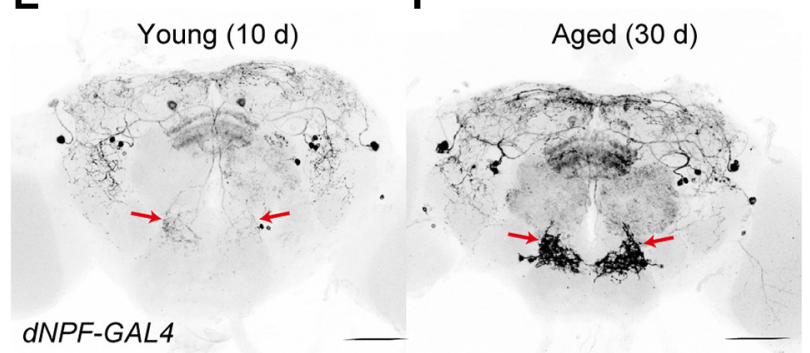

B

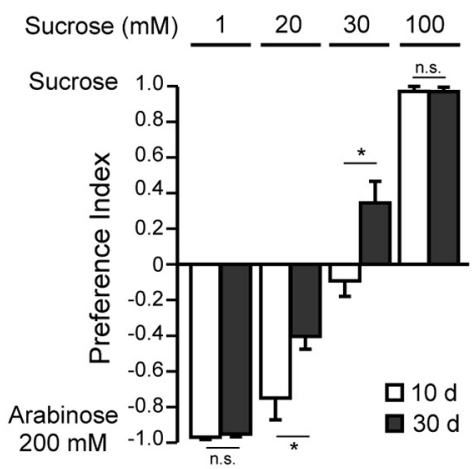

C

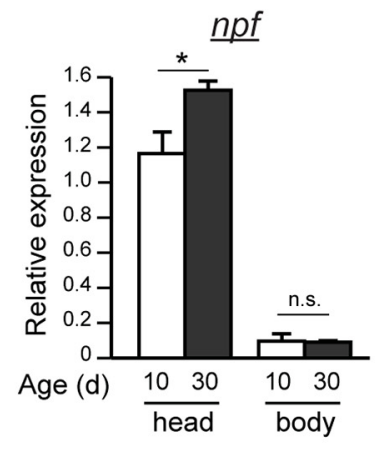

D

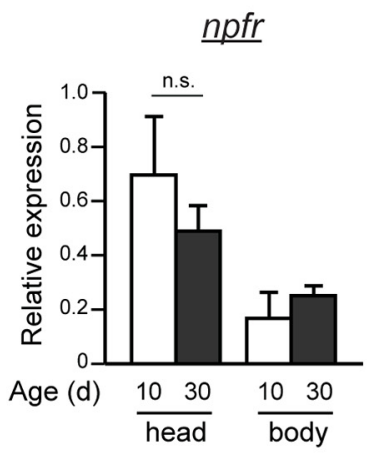

G

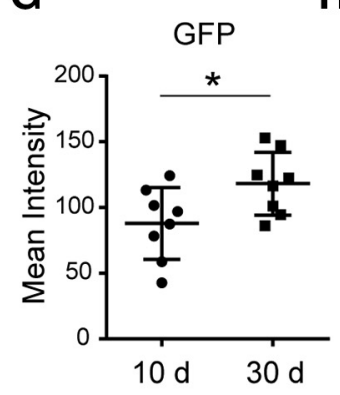

H

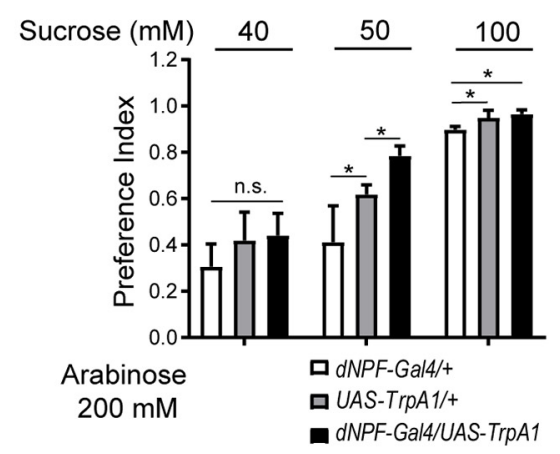

Figure 3. Aging modulates $d N P F$ pathway regulating nutritious sugar preference. $\boldsymbol{A}$, Two-choice preference assay. Flies are given a choice between two food substrates, colored with tasteless food dyes, red or blue. The food preference for each fly is scored by examining the color of the abdomen, red, blue, or purple, which means that a fly chose both foods. $\boldsymbol{B}$, Food preferences in the two-choice assay (1, 20, 30, or $100 \mathrm{~mm}$ sucrose vs $200 \mathrm{~mm}$ arabinose) in (anton-S flies at 10 or $30 \mathrm{~d}$ of age, following food deprivation. Aged flies show a stronger preference for nutritious sugar over non-nutritious sugar compared with young flies, when 20 or $30 \mathrm{~mm}$ sucrose was used. $n=4,4,3$, and 4 for sucrose and $1 \mathrm{~mm}, 20 \mathrm{~mm}$, $30 \mathrm{~mm}$, and $100 \mathrm{~mm}$ data, respectively. Data are shown as mean \pm SD. C, D, dNPF expression is significantly increased in fly heads in 30-d-old flies compared with 10-d-old flies. $\boldsymbol{C}$, No significant difference was observed in expression of $d N P F R$. $\boldsymbol{D}, n=3$ for each. n.S.: not significant. Data are shown as mean \pm SD. $\boldsymbol{E}, \boldsymbol{F}$, Fly brains expressing $m$ CD8::GFP driven by dNPF-Gal 4 at $10 \mathrm{~d}$ of age $\boldsymbol{E}$, and at $30 \mathrm{~d}$ of age. $\boldsymbol{F}$, Expression of GFP is higher in aged compared with young flies, especially in the SOG area (red arrows). $\mathbf{G}$, Quantification of GFP expression in the SOG area (E,F). Mean intensity of GFP expression in the SOG area in flies expressing $m$ CD8::GFP driven by dNPF-Gal4. $n=8$ for each. $\boldsymbol{H}$, Food preferences in the two-choice assay (40,50, or 100 mm sucrose vs 200 mm arabinose) in dNPF-Gal4 driven TrpA1 expressing flies (dNPF-Gal4/UAS-TrpA1), UAS-TrpA1 control flies (UAS-TrpA1/+), or dNPF-Gal4 control flies (dNPF-Gal4/+), at $10 \mathrm{~d}$ of age after food deprivation. The assay was performed at $31^{\circ} \mathrm{C}$. Flies with activation of $d N P F$ expressing cells show stronger preference to nutritious sugar over non-nutritious sugar compared with UAS-TrpA1 or dNPF-Gal4 control flies. $n=5,6$, and 5 for 40 mM $-d N P F-G a l 4 /+, U A S-T r p A 1 /+$, and dNPF-Gal4>UAS-TrpA1. $n=4,7$, and 4 for $50 \mathrm{~mm}-d N P F-G a l 4 /+$, UAS-TrpA1/+, and dNPF-Gal4/UAS-TrpA1. $n=4,8$, and 4 for $100 \mathrm{~mm}-d N P F-G a l 4 /+$, UAS-TrpA1/+, and dNPF-Gal4/UASTrpA1. Data are shown as mean \pm SD.

GFP construct in adult fly brains, revealed neurons in the subesophageal ganglion (SOG), the dorsal and lateral protocerebrum, and the central complex (Fig. 3E). We compared the $d N P F-G a l 4$-labeled GFP between young and aged flies and found that the intensity of $d N P F$ expression was upregulated specifically around the SOG area in aged flies $\left(t_{(14)}=2.359,{ }^{\star} p=0.0334\right.$, unpaired $t$ test) (Fig. $3 E-G$ ), suggesting an age-dependent increase in $d N P F$ expression.

To examine whether the enhanced expression or activation of $d N P F$-expressing neurons in aged flies causes an increased preference for nutritious sugar over non-nutritious sugar, we expressed the heat-sensitive uas-TrpA1 transgene with $d N P F-G a l 4$ and measured sugar preferences in flies at $10 \mathrm{~d}$ of age. Ectopically expressed TrpA1 depolarizes neurons when flies are exposed to temperatures $>25^{\circ} \mathrm{C}$ (Hamada et al., 2008). A two-choice assay was performed in flies with activation of the $d N P F$-expressing neurons by exposing the flies to $31^{\circ} \mathrm{C}$ to examine the preference for the nutritious sugar sucrose over a concentrated nonnutritious sugar arabinose. Flies with activated $d N P F$-expressing neurons ( $d N P F-G a l 4 / U A S$-TrpA1) showed higher preference for the less concentrated nutritious sugar over a highly concentrated non-nutritious sugar compared with the control flies not expressing TrpA1 (dNPF-Gal4/+ or UAS-TrpA1/+) (one-way
ANOVA followed by post hoc Tukey's multiple-comparisons test. $40 \mathrm{~mm}: F_{(2,13)}=2.332, p=0.1363 ; 50 \mathrm{~mm}: F_{(2,12)}=18.82, p=$ 0.0002 , post hoc Tukey's multiple-comparisons test, $p=0.0001$, $d N P F-G a l 4 /+$ vs dNPF-Gal4/UAS-TrpA1, $p=0.0239$, UAS$\operatorname{TrpA1/+}$ vs dNPF-Gal4/UAS-TrpA1, $p=0.0064, d N P F-G a l 4 /+$ vs $U A S-T r p A 1 /+; 100 \mathrm{~mm}: F_{(2,13)}=7.605, p=0.0065$, post hoc Tukey's multiple-comparisons test, $p=0.0073, d N P F-G a l 4 /+$ vs $d N P F-G a l 4 / U A S-T r p A 1, p=0.5544, U A S-T r p A 1 /+$ vs $d N P F-$ Gal4/UAS-TrpA1, $p=0.0183, d N P F-G a l 4 /+$ vs $U A S-T r p A 1 /+)$ (Fig. $3 H$ ). These data indicate that the activation of $d N P F-$ expressing neurons induces a higher preference for nutritious sugar over non-nutritious sugar.

\section{Age-dependent effect on dopaminergic neurons conveying reward signals of sweetness}

The recent studies indicate that the PAM cluster of DANs innervating the horizontal MB lobes relays reward signals (Huetteroth et al., 2015; Yamagata et al., 2015; Kaun and Rothenfluh, 2017). The PAM cluster of DANs labeled with R48B04-Gal4 and projecting to two different lobes of the mushroom bodies, $\beta$ ' 2 and $\gamma 4$ (PAM- $\gamma 4)$, is involved in conveying the sweetness of sugar for STM. Another PAM cluster of DANs labeled with R15A04-Gal4 and projecting to the base of the $\alpha$ lobe (PAM- $\alpha 1)$ is responsible 
A

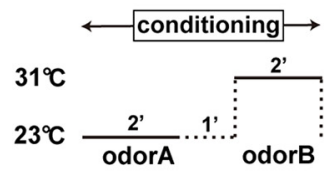

B
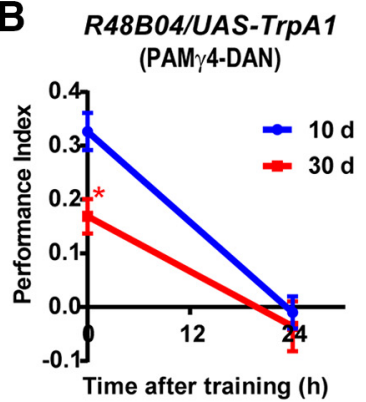

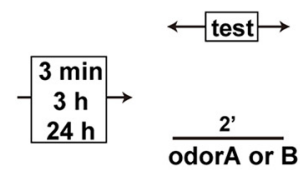

C R15A04/UAS-TrPA1 (PAM $\alpha 1$-DAN)

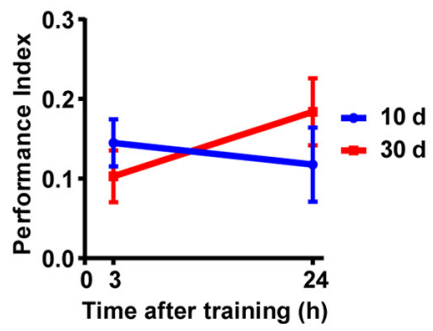

D

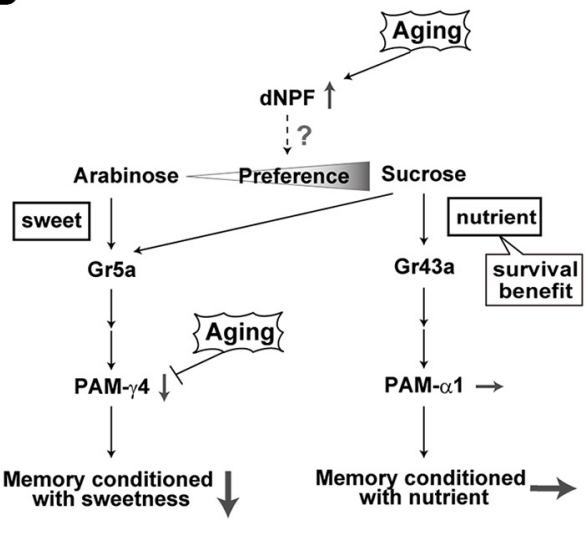

Figure 4. Age-dependent effect on DANs conveying reward signals of sweetness. A, Experimental protocol. Flies with thermoactivation paired with an odor presentation for artificial conditioning are tested $3 \mathrm{~min}, 3 \mathrm{~h}$, or $24 \mathrm{~h}$ after conditioning. B, Appetitive short-term-memory ( $3 \mathrm{~min}$ after conditioning) formed by thermoactivation paired with an odor presentation in R48B04-Gal4/UAS-TrpA1 flies is decreased in 30-d-old flies compared with 10-d-old flies. However, appetitive long-term-memory (24 h after conditioning) is not formed both in 10 and 30-d-old flies. $n=9,16,6$, and 6 for $10 \mathrm{~d}$ to $0 \mathrm{~h}, 30 \mathrm{~d}$ to $0 \mathrm{~h}, 10 \mathrm{~d}$ to $24 \mathrm{~h}$, and $30 \mathrm{~d}$ to $24 \mathrm{~h}$ data, respectively. Data are shown as mean \pm SEM. C, Appetitive long-term-memory formed by thermoactivation paired with an odor presentation in R15A04-Ga/4/UAS-TrpA1 flies is not changed across aging. $n=9,10,12$, and 8 for $10 \mathrm{~d}$ to $3 \mathrm{~h}, 30 \mathrm{~d}$ to $3 \mathrm{~h}, 10 \mathrm{~d}$ to $24 \mathrm{~h}$, and $30 \mathrm{~d}$ to $24 \mathrm{~h}$ data, respectively. Data are shown as mean \pm SEM. $D$, Model of the age-dependent effect on appetitive memory in the fly brain. Properties of sweetness and nutrition are independently conveyed to distinct clusters of the PAM DANs. Aging modulates the dNPF pathway regulating nutritious sugar preference and impairs the function of PAM- $\gamma 4$ DANs conveying sweetness of sugar. These age-dependent changes lead to fragile appetitive memory with the non-nutritious sugar arabinose, but robust appetitive memory associated with the nutritious sugar sucrose.

for conveying nutritious signals of sugar for LTM. We next examined how aging affects the function of PAM- $\gamma 4$ DANs conveying the sweetness of sugar and the function of PAM- $\alpha 1$ conveying nutritious signals of sugar.

We activated the PAM- $\gamma 4$ DANs by thermogenetic activation with $\operatorname{Trp} A 1$ at the time of an odor presentation and compared this artificial formation of appetitive memory at $3 \mathrm{~min}$ or $24 \mathrm{~h}$ after conditioning in young and aged flies (Fig. 4A). As previously shown, the thermoactivation of PAM- $\gamma 4$ DANs paired with an odor presentation in R48B04-Gal4/UAS-TrpA1 flies after starvation for $40 \mathrm{~h}$ induced memory formation immediately after artificial conditioning in young flies $(0 \mathrm{~h}$ in Fig. $4 B$ ) but not LTM formation ( $24 \mathrm{~h}$ in Fig. $4 B$ ). In contrast, aging significantly decreases memory formation induced by activation of PAM- $\gamma 4$ DANs (two-way ANOVA followed by post hoc Tukey's multiplecomparisons test. $F_{(1,33)}=5.133, p=0.0302$, post hoc Tukey's multiple-comparisons test, $0 \mathrm{~h}:{ }^{\star} p=0.0109,10 \mathrm{~d}$ vs $30 \mathrm{~d} ; 24 \mathrm{~h}$ : $p=0.9796,10 \mathrm{~d}$ vs $30 \mathrm{~d})(0 \mathrm{~h}$ in Fig. $4 B$ ). We next activated the PAM- $\alpha 1$ by thermogenetic activation with $\operatorname{Tr} A A 1$ at the time of an odor presentation and compared the artificial formation of appetitive memory at 3 and $24 \mathrm{~h}$ after conditioning in young and aged flies (Fig. 4A). Aging did not affect memory formation induced by the activation of PAM- $\alpha 1$ DANs (two-way ANOVA followed by post hoc Tukey's multiple-comparisons test. $F_{(1,35)}=$ 0.09032, $p=0.7655$ ) (Fig. $4 C$ ). These data suggest that aging impairs the function of PAM- $\gamma 4$ DANs, leading to age-dependent impairment in memory formation conditioned with sweetness, such as arabinose; however, aging does not affect the function of PAM- $\alpha 1$ DANs, leading to the robust formation of memory conditioned with nutrient across aging.

\section{Discussion}

We have provided several lines of evidence indicating that appetitive memory is formed robustly in aged flies when they are conditioned with nutritious sugar that increases their survival benefit in a starved condition (Fig. 4D). The robustness of appetitive memory conditioned with nutritious sugar in Drosophila is consistent with a previous study showing that reward memory remains intact during aging in mice (Harb et al., 2014). Various studies suggest that postingestive mechanisms allow animals to sense the nutrient value of sugars. For example, animals carrying mutations for sensing sugar taste develop a preference for sugar nutrients independently from sweet taste inputs, in both flies and mice (de Araujo et al., 2008; Dus et al., 2011). Our results suggest that aging does not affect the system that senses the nutrient value of sugars. The nutrient property is conveyed through Gr43a neurons to PAM- $\alpha_{1}$ DANs, projecting to the $\alpha_{1}$ regions of the mushroom body lobes (Miyamoto et al., 2012). Our results of the thermogenetic experiment (Fig. 4C) indicate that aging preserves memory formation after conditioning with nutritious sugar, with odor presentation, suggesting the intact functioning of Gr43a neurons and PAM- $\alpha_{1}$ DANs in aged flies. However, there remains the possibility that conditioning associated with nutrition intake in the starved condition covers the energy requirement to form appetitive LTM (Plaçais and Preat, 2013; Plaçais et al., 2017); hence, aged flies form robust appetitive memory with nutritious sugar, but not with non-nutritious sugar.

When flies were conditioned with non-nutritious sugar without survival benefits, the formation of appetitive memory is fragile in aged flies. The sweetness of sugar is perceived by Gr5a neurons and conveyed through octopaminergic neurons (OANs) to PAM- $\gamma_{4}$ DANs, expressing the OAMB octopamine receptor (Han et al., 1998) and projecting to the $\gamma_{4}$ regions of the MB lobes (Huetteroth et al., 2015; Yamagata et al., 2015). Several possible mechanisms exist for the age-dependent impairment of appetitive memory conditioned with arabinose, such as the following: (1) aging impairs the activity of Gr5a expressing neurons responding to sugars, (2) the function of OANs is impaired in aged flies, and (3) the function of PAM- $\gamma_{4}$ DANs is affected by age. According to our PER assay data, the sensitivity to sucrose or arabinose under the starved condition was not changed with ag- 
ing, suggesting that Gr5a neurons signal sweetness in aged flies similar to young flies. Although whether aging affects OANs was unclear from this study, our results showed that an implanted artificial appetitive memory in young flies by thermogenetic activation of the PAM- $\gamma_{4}$ DANs with odor presentation was impaired in aged flies (Fig. $4 B$ ), suggesting an age-dependent impairment in the function of PAM- $\gamma_{4}$ DANs or other downstream mechanisms. Several studies have shown an age-dependent impairment of DANs (Li and Rieckmann, 2014). Because our data showed that aging affects the function of PAM- $\gamma 4$ DANs, but not PAM- $\alpha 1$ DANs, our study demonstrated that aging has different effects on the distinct types of DANs.

The previous studies have shown that aging decreases intermediateterm memory formation after single-cycle aversive conditioning, but not STM (Tamura et al., 2003; Tonoki and Davis, 2012). Negative reinforcement signals for acquisition of aversive STM are predominantly carried by protocerebral posterior lateral (PPL) DANs (Claridge-Chang et al., 2009; Mao and Davis, 2009; Aso et al., 2010, 2012). The formation of normal aversive STM in aged flies suggests consistent function of PPL DANs across aging, further supporting the age-dependent distinct effects on DAN types. Memory retention after aversive conditioning is suppressed in aged flies, leading to decreased intermediate-term memory, partly due to the dysfunction of the dorsal paired medial (DPM) neurons (Tamura et al., 2003; Tonoki and Davis, 2012), which are required during the interval between conditioning and testing for both aversive and appetitive memory formations (Waddell et al., 2000; Keene et al., 2006; Cervantes-Sandoval and Davis, 2012). Our observations suggest that synaptic transmission from the DPM neurons is observed in aged flies when they are conditioned with nutritious sugar in a starved condition.

Motivation is a key factor in appetitive learning and eating behavior (Dayan and Balleine, 2002; Kringelbach et al., 2012). The previous studies have shown that $d N P F$ expressing neurons increase sugar sensitivity (Inagaki et al., 2014) and promote appetitive memory performance (Krashes et al., 2009). Our studies showed that aging increases $d N P F$ expression, while the expression of $d N P F$ is possibly upregulated by a homeostatic response due to age-related reduced dNPF peptide level. Furthermore, artificial activation of $d N P F$-expressing neurons using thermogenetics enhances preference for nutritious sugar over nonnutritious sugar. These data suggest that age-dependent increase in $d N P F$ expression may enhance the preference for nutritious sugar over non-nutritious sugar, possibly through the enhanced activation of Gr43a neurons, to convey nutrient signals through the higher expression of $d N P F$.

\section{References}

Aso Y, Herb A, Ogueta M, Siwanowicz I, Templier T, Friedrich AB, Ito K, Scholz H, Tanimoto H (2012) Three dopamine pathways induce aversive odor memories with different stability. PLoS Genet 8:e1002768.

Aso Y, Siwanowicz I, Bräcker L, Ito K, Kitamoto T, Tanimoto H (2010) Specific dopaminergic neurons for the formation of labile aversive memory. Curr Biol 20:1445-1451.

Aso Y, Sitaraman D, Ichinose T, Kaun KR, Vogt K, Belliart-Guérin G, Plaçais PY, Robie AA, Yamagata N, Schnaitmann C, Rowell WJ, Johnston RM, Ngo TT, Chen N, Korff W, Nitabach MN, Heberlein U, Preat T, Branson KM, Tanimoto H, et al. (2014a) Mushroom body output neurons encode valence and guide memory-based action selection in Drosophila. Elife 3:e04580.

Aso Y, Hattori D, Yu Y, Johnston RM, Iyer NA, Ngo TT, Dionne H, Abbott LF, Axel R, Tanimoto H, Rubin GM (2014b) The neuronal architecture of the mushroom body provides a logic for associative learning. Elife 3:e04577.
Burke CJ, Waddell S (2011) Remembering nutrient quality of sugar in Drosophila. Curr Biol 21:746-750.

Cervantes-Sandoval I, Davis RL (2012) Distinct traces for appetitive vs aversive olfactory memories in DPM neurons of Drosophila. Curr Biol 22:1247-1252.

Claridge-Chang A, Roorda RD, Vrontou E, Sjulson L, Li H, Hirsh J, Miesenböc G (2009) Writing memories with light-addressable reinforcement circuitry. Cell 139:405-415.

Dayan P, Balleine BW (2002) Reward, motivation, and reinforcement learning. Neuron 36:285-298.

de Araujo IE, Oliveira-Maia AJ, Sotnikova TD, Gainetdinov RR, Caron MG, Nicolelis MA, Simon SA (2008) Food reward in the absence of taste receptor signaling. Neuron 57:930-941.

Dus M, Min S, Keene AC, Lee GY, Suh GS (2011) Taste-independent detection of the caloric content of sugar in Drosophila. Proc Natl Acad Sci U S A 108:11644-11649.

Fujita M, Tanimura T (2011) Drosophila evaluates and learns the nutritional value of sugars. Curr Biol 21:751-755.

Hamada FN, Rosenzweig M, Kang K, Pulver SR, Ghezzi A, Jegla TJ, Garrity PA (2008) An internal thermal sensor controlling temperature preference in Drosophila. Nature 454:217-220.

Han KA, Millar NS, Davis RL (1998) A novel octopamine receptor with preferential expression in Drosophila mushroom bodies. J Neurosci 18: $3650-3658$.

Harb MR, Sousa N, Zihl J, Almeida OF (2014) Reward components of feeding behavior are preserved during mouse aging. Front Aging Neurosci 6:242.

Huetteroth W, Perisse E, Lin S, Klappenbach M, Burke C, Waddell S (2015) Sweet taste and nutrient value subdivide rewarding dopaminergic neurons in Drosophila. Curr Biol 25:751-758.

Inagaki HK, Ben-Tabou de-Leon S, Wong AM, Jagadish S, Ishimoto H, Barnea G, Kitamoto T, Axel R, Anderson DJ (2012) Visualizing neuromodulation in vivo: TANGO-mapping of dopamine signaling reveals appetite control of sugar sensing. Cell 148:583-595.

Inagaki HK, Panse KM, Anderson DJ (2014) Independent, reciprocal neuromodulatory control of sweet and bitter taste sensitivity during starvation in Drosophila. Neuron 84:806-820.

Kaun KR, Rothenfluh A (2017) Dopaminergic rules of engagement for memory in Drosophila. Curr Opin Neurobiol 43:56-62.

Keene AC, Krashes MJ, Leung B, Bernard JA, Waddell S (2006) Drosophila dorsal paired medial neurons provide a general mechanism for memory consolidation. Curr Biol 16:1524-1530.

Krashes MJ, Waddell S (2008) Rapid consolidation to a radish and protein synthesis-dependent long-term memory after single-session appetitive olfactory conditioning in Drosophila. J Neurosci 28:3103-3113.

Krashes MJ, DasGupta S, Vreede A, White B, Armstrong JD, Waddell S (2009) A neural circuit mechanism integrating motivational state with memory expression in Drosophila. Cell 139:416-427.

Kringelbach ML, Stein A, van Hartevelt TJ (2012) The functional human neuroanatomy of food pleasure cycles. Physiol Behav 106:307-316.

Lee T, Luo L (1999) Mosaic analysis with a repressible cell marker for studies of gene function in neuronal morphogenesis. Neuron 22:451461.

Li SC, Rieckmann A (2014) Neuromodulation and aging: implications of aging neuronal gain control on cognition. Curr Opin Neurobiol 29:148 158.

Lin YJ, Seroude L, Benzer S (1998) Extended life-span and stress resistance in the Drosophila mutant methuselah. Science 282:943-946.

Mao Z, Davis RL (2009) Eight different types of dopaminergic neurons innervate the Drosophila mushroom body neuropil: anatomical and physiological heterogeneity. Front Neural Circuits 3:5.

Mery F (2007) Aging and its differential effects on consolidated memory forms in Drosophila. Exp Gerontol 42:99-101.

Miyamoto T, Slone J, Song X, Amrein H (2012) A fructose receptor functions as a nutrient sensor in the Drosophila brain. Cell 151:11131125.

Plaçais PY, Preat T (2013) To favor survival under food shortage, the brain disables costly memory. Science 339:440-442.

Plaçais PY, de Tredern É, Scheunemann L, Trannoy S, Goguel V, Han KA, Isabel G, Preat T (2017) Upregulated energy metabolism in the Drosophila mushroom body is the trigger for long-term memory. Nat Commun 8:15510. 
Sahu A, Kalra PS, Kalra SP (1988) Food deprivation and ingestion induce reciprocal changes in neuropeptide $\mathrm{Y}$ concentrations in the paraventricular nucleus. Peptides 9:83-86.

Tamura T, Chiang AS, Ito N, Liu HP, Horiuchi J, Tully T, Saitoe M (2003) Aging specifically impairs amnesiac-dependent memory in Drosophila. Neuron 40:1003-1011.

Tanimura T, Isono K, Yamamoto MT (1988) Taste sensitivity to trehalose and its alteration by gene dosage in Drosophila melanogaster. Genetics 119:399-406.

Tempel BL, Bonini N, Dawson DR, Quinn WG (1983) Reward learning in normal and mutant Drosophila. Proc Natl Acad Sci U S A 80:1482-1486.

Tonoki A, Davis RL (2012) Aging impairs intermediate-term behavioral memory by disrupting the dorsal paired medial neuron memory trace. Proc Natl Acad Sci U S A 109:6319-6324.
Tonoki A, Davis RL (2015) Aging impairs protein-synthesis-dependent long-term memory in Drosophila. J Neurosci 35:1173-1180.

Tully T, Preat T, Boynton SC, Del Vecchio M (1994) Genetic dissection of consolidated memory in Drosophila. Cell 79:35-47.

Waddell S, Armstrong JD, Kitamoto T, Kaiser K, Quinn WG (2000) The amnesiac gene product is expressed in two neurons in the Drosophila brain that are critical for memory. Cell 103:805-813.

Wen T, Parrish CA, Xu D, Wu Q, Shen P (2005) Drosophila neuropeptide F and its receptor, NPFR1, define a signaling pathway that acutely modulates alcohol sensitivity. Proc Natl Acad Sci U S A 102:2141-2146.

Yamagata N, Ichinose T, Aso Y, Plaçais PY, Friedrich AB, Sima RJ, Preat T, Rubin GM, Tanimoto H (2015) Distinct dopamine neurons mediate reward signals for short- and long-term memories. Proc Natl Acad Sci U S A 112:578-583. 